FORMATION Formation emploi

Revue française de sciences sociales

145 | Janvier-Mars 2019

Varia

\title{
Les trajectoires socialement et spatialement différenciées des étudiants franciliens
}

Socially and spatially distinguished academic trajectories of students in Paris urban area

Soziale und räumliche Unterschiede zwischen den Studienwegen in der Ile-deFrance

Las trayectorias social y espacialmente diferenciadas de los estudiantes de Ilede-France

\section{Leïla Frouillou et Léonard Moulin}

\section{(2) OpenEdition} Journals

Édition électronique

URL : https://journals.openedition.org/formationemploi/6845

DOI : 10.4000/formationemploi.6845

ISSN : 2107-0946

Éditeur

La Documentation française

Édition imprimée

Date de publication : 15 avril 2019

Pagination : 7-28

ISSN : 0759-6340

Référence électronique

Leilla Frouillou et Léonard Moulin, « Les trajectoires socialement et spatialement différenciées des étudiants franciliens », Formation emploi [En ligne], 145 | Janvier-Mars 2019, mis en ligne le 01 janvier 2021, consulté le 03 septembre 2021. URL : http://journals.openedition.org/formationemploi/6845 ; DOI : https://doi.org/10.4000/formationemploi.6845 


\title{
Les trajectoires socialement et spatialement différenciées des étudiants franciliens
}

\author{
LEÏLA FROUILLOU \\ Maîtresse de conférences en sociologie, à I'université Paris Nanterre, Centre de recherches \\ sociologiques et politiques de Paris, équipe Genre-Travail-Mobilité \\ LÉONARD MOULIN \\ Économiste, chargé de recherche à I'INED (Institut national d'études démographiques)
}

Résumé

\begin{abstract}
Les trajectoires socialement et spatialement différenciées des étudiants franciliens

En croisant un suivi quantitatif (analyse de séquences) et qualitatif (entretiens répétés) d'étudiants en droit et en AES (administration économique et sociale) en région francilienne, cet article montre que les trajectoires universitaires diffèrent selon la discipline et l'établissement d'inscription. La typologie souligne la prépondérance des trajectoires stables (même université). Les trajectoires de sortie du système universitaire se différencient quant à elles selon la discipline et le lieu d'études (Paris/banlieue). Enfin, les trajectoires interuniversitaires, minoritaires, révèlent un " sens du placement " étudiant qui contribue à attirer les étudiants les mieux dotés vers les universités les plus favorisées.
\end{abstract}

Mots clés : enseignement superieur, étudiant, cheminement universitair, origine sociale, origine scolaire

Abstract

Socially and spatially distinguished academic trajectories of students in Paris urban area

Intersecting quantitative analysis by optimal matching (sequencing) with multiple qualitative interviews with students in Law or AES (French academic courses including social administration and mainly targeting professional and technological students) in and around Paris, this article shows how academic courses vary according to fields of study and chosen institutions. Typology emphasizes the prevalence of stable courses (unchanging university), whereas scholar-escaping tracks are mainly explained according to field of study and geography (Paris/suburban area). Lastly, interacade- 
mical courses, which are few, disclose a "positioning insight" on behalf of students who tend to appeal to best universities because of their upscale background.

Keywords: higher education; student; undergraduate paths; social origin; former schooling

Journal of Economic Literature: I 23

Traduction : Auteur.e.s

L'Île-de-France compte dix-sept universités ${ }^{1}$, caractérisées par des logiques concurrentielles et des complémentarités (Baron \& al., 2007), selon un fonctionnement systémique. Celui-ci différencie les établissements selon plusieurs critères (orientation disciplinaire, nombre d'étudiants, poids des cycles d'étude), et notamment en fonction de l'origine sociale et des parcours scolaires.

Plusieurs facteurs expliquent ces différenciations de publics : la localisation des établissements dans une agglomération ségrégée et inégalement desservie en transports en commun, les politiques d'établissement ou encore le fonctionnement des systèmes d'affectation à l'entrée en licence 1 (L1).

Cet espace universitaire francilien et ses différenciations en termes de publics étudiants peuvent être saisis à partir des trajectoires étudiantes qui dessinent des polarités symboliques entre universités. En outre, l'approche longitudinale contribue à déconstruire une lecture normative trop linéaire des parcours universitaires (Bodin, Orange, 2013).

Cet article propose de lire de manière dynamique ces différenciations de publics entre universités franciliennes, en considérant les trajectoires d'étudiants inscrits en droit et AES (administration économique et sociale), entre 2007 et 2011. Ces deux filières ont été choisies car elles présentent une certaine proximité dans les enseignements, tout en se caractérisant par des populations étudiantes socialement et scolairement distinctes. L'AES est en effet une filière où les bacheliers professionnels et technologiques sont surreprésentés et qui a été pensée, dès sa création, comme une filière professionnalisante de l'enseignement supérieur (Lefeuvre, 2011). Cela permet de tenir compte de la hiérarchie disciplinaire.

Dans la perspective de nombreux travaux sur les inégalités sociales dans les trajectoires d'études, autour de la sélection sociale ${ }^{2}$ opérée par le système scolaire, les décrochages et

1. L’université Paris 9 Dauphine a le statut de Grand Établissement depuis 2004.

2. À l'échelle nationale, «la poursuite d'études longues à l'université est plus le fait de jeunes dont les parents sont cadres supérieurs ou exerçant une profession libérale : leur part passe de $28 \%$ en cursus licence à $34 \%$ en cursus doctorat. Inversement, alors que les enfants d'ouvriers représentent $13 \%$ des étudiants inscrits à l'université en cursus Licence, leur part est de $5 \%$ en cursus doctorat " (DEPP, 2015, p. 188). Cette sélection est aussi scolaire : les trois quarts des bacheliers technologiques entrés à l'Université en 2008 ne poursuivent plus d'études en 2013, la moitié n'ayant obtenu aucun diplôme. 
les réorientations (Bourdieu \& Passeron, 1964 ; Bodin \&Millet, 2011 ; Sarfati, 2015), notre première hypothèse est que la sélection est plus forte dans les établissements où les publics de L1 sont les plus défavorisés. Cette sélection différentielle serait avant tout scolaire, comme l'a montré une récente étude sur les étudiants de la filière AES : "Les étudiants les plus dépourvus des prérequis attendus par l'université ont en grande partie disparu [entre la L1 et la L3]» (Nicourd \& al., 2013, p. 9).

Notre seconde hypothèse est que les étudiants changeant d'université possèdent des caractéristiques sociales et scolaires qui s'écartent de celles des " sédentaires ». Ces trajectoires interuniversitaires traduiraient un "sens du placement " étudiant (Frouillou, 2015), autrement dit un ajustement social et scolaire à la filière et à l'établissement.

Pour vérifier ces deux hypothèses, nous croisons une méthode d'appariement optimal isolant des types de trajectoires avec une enquête par entretiens auprès d'étudiants. Ces types de trajectoires sont replacés dans les trajectoires familiales et scolaires des enquêtés, pour interroger l'apprentissage des dispositions à s'orienter au fil des cursus.

Dans une première partie, nous faisons état des travaux utilisant l'approche longitudinale ou portant sur les universités franciliennes. La deuxième partie présente les données, la méthode, ainsi que les typologies des trajectoires universitaires, construites grâce à l'optimal matching. La troisième partie est quant à elle consacrée aux deux types de trajectoires majoritaires : " rester dans la même université " et "sortir du système universitaire ». La quatrième partie enfin s'intéresse aux trajectoires « interuniversitaires ».

\section{1 " Réintroduire le temps »: une approche par les trajectoires}

Au croisement de la littérature sur les trajectoires scolaires et sur les universités franciliennes, cet article interroge les trajectoires étudiantes à l'échelle des établissements universitaires franciliens.

Nous retenons le cadre d'analyse bourdieusien pour « saisir la structuration des biographies à la fois comme un effet des structurations longitudinales qui se résument en amont dans 'linstitution biographique' et comme le produit agrégé que l'action sociale des individus inscrit, en aval, dans le maintien ou la transformation de ces structures longitudinales" (ibid., p. 20).

Cette approche permet d'étudier les " trajectoires étudiantes " comme élément d'analyse des modes de reproduction sociale. Les trajectoires sont définies comme la "série des positions successivement occupées par un même agent (ou un même groupe) dans un espace luimême en devenir et soumis à d'incessantes transformations. [...] Les événements biographiques se définissent comme autant de placements et de déplacements dans l'espace social, c'est-à-dire, plus précisément, dans les différents états successifs de la structure de la distribution des différentes espèces de capital qui sont en jeu dans le champ considéré" (Bourdieu, 1994, p. 88). 
Les données longitudinales sont centrales dans l'appréhension et la compréhension des inégalités sociales reproduites par l'institution scolaire car elles sont cumulatives (Duru-Bellat, 2002).

Les recherches sur l'enseignement supérieur mobilisent fréquemment l'entrée par les parcours ou les trajectoires pour appréhender les choix de filières, la réussite, le décrochage et l'insertion professionnelle (Beaupère \& Boudesseul, 2009 ; Borras \& al., 2012 ; Cordazzo, 2014 ; Erlich \& Verley, 2010 ; Hugrée, 201 ; Nicourd \& al., 2011 ; Orange, 2013 ; Truong, 2015). Néanmoins, les recherches s'appuyant sur des cohortes ou des panels d'étudiants sont encore rares. Pourtant, cette approche permet de saisir les temporalités de déclenchement des trajectoires interuniversitaires, les réorientations disciplinaires ou encore les reprises d'études, tout en considérant l'origine sociale et scolaire des étudiants.

Le paysage universitaire francilien a fait l'objet de travaux historiques, sociologiques et géographiques mettant en évidence ses héritages symboliques (Girault \& al., 2012), son offre de formation et les recrutements étudiants associés (De Berny, 2008 ; Frouillou, op. cit.) et son fonctionnement systémique (Baron \& al., 2007 ; Berroir \& al., 2005). Ces recherches montrent le caractère d'isolat de celui-ci (les mobilités étudiantes en font un sous-système), qui en fait un terrain d'études propice aux analyses de trajectoires.

Cependant, les analyses fondées sur la comparaison des trajectoires étudiantes dans différentes universités franciliennes sont peu développées, hormis les travaux quantitatifs et qualitatifs menés par Nicourd \& al. $(2011 ; 2013)$.

\section{Explorer
les trajectoires étudiantes}

Cette partie expose les données et méthodes permettant de construire des types de trajectoires pour les étudiants de droit et d'AES.

\subsection{Données et méthodes pour construire une typologie}

Les données ${ }^{3}$ utilisées résultent d'un appariement entre les bases SISE universités (Système d'information sur le suivi de l'étudiant) et les bases AGLAE (Application de gestion du logement et de l'aide à l'étudiant) du MESR (ministère de l'Enseignement supérieur et de la Recherche).

3. Les analyses ont été réalisées à partir des bases de données SISE universités (inscriptions et résultats) et AGLAE - MESR DGESIP-DGRI-SIES / système d'information SISE et système d'information AGLAE (extraction au 15 mars 2013). 
L’origine sociale des étudiants est appréhendée à partir de la PCS (Profession et catégorie socioprofessionnelle) du " chef de famille ». Le fait de ne disposer que de la PCS d'un seul membre du ménage constitue une limite importante, mais fournit une approximation de l'origine sociale des étudiants. Cette variable est ensuite agrégée en quatre catégories, de "très favorisée " à " défavorisée " (voir tableau A2), en suivant le regroupement proposé par la DEPP (Direction de l'évaluation, de la prospective et de la performance). Outre sa facilité d'utilisation, ce regroupement permet de fournir un cadre d'analyse sociologique de la position sociale des étudiants ${ }^{4}$ et de réduire les effets de la qualité moyenne de l'information recueillie (Merle, 2013). L'origine géographique utilisée dans cet article fait référence au département de résidence des parents.

Les méthodes d'appariement optimal (Lesnard \& De Saint Pol 2006) reposent sur une mesure de distance entre trajectoires pour en déduire une typologie. La comparaison des trajectoires s'appuie sur la retranscription de celles-ci sous forme de séquences (ou listes ordonnées) d'états. Chaque état correspond à une situation dans laquelle se trouve un individu à un moment donné.

4. Pour une partie des étudiants primo-inscrits en 2007, la PCS du " chef de famille " n'était pas renseignée lors de leur première inscription ( $7 \%$ en AES et $10 \%$ en droit). Nous avons tenté de récupérer cette information en utilisant l'information disponible les années suivantes. Cela nous a permis de réduire la part de PCS non renseignée ( $3 \%$ en AES et $4 \%$ en droit). Cette part étant inférieure à $5 \%$, une imputation permettant de corriger les valeurs manquantes a alors été réalisée en tenant compte de la distribution des valeurs réellement observées pour chaque discipline. 
Tableau A2 : Regroupement des PCS

(professions et catégories socioprofessionnelles)

\begin{tabular}{|c|c|}
\hline Catégorie & PCS \\
\hline Très favorisée & $\begin{array}{l}\text { Chefs d'entreprise de dix salariés ou plus } \\
\text { Professions libérales } \\
\text { Cadres de la fonction publique } \\
\text { Professeurs et assimilés } \\
\text { Professions de l'information, des arts et du spectacle } \\
\text { Cadres administratifs et commerciaux d'entreprise } \\
\text { Ingénieurs, cadres techniques d'entreprise } \\
\text { Instituteurs et assimilés }\end{array}$ \\
\hline Favorisée & $\begin{array}{l}\text { Professions intermédiaires de la santé et du travail social } \\
\text { Clergé } \\
\text { Professions intermédiaires administratives de la fonction publique } \\
\text { Professions intermédiaires administratives du commerce ou des entreprises } \\
\text { Techniciens } \\
\text { Contremaîtres, agents de maîtrise } \\
\text { Retraités cadres et professions intermédiaires }\end{array}$ \\
\hline Moyenne & $\begin{array}{l}\text { Agriculteurs exploitants } \\
\text { Artisans } \\
\text { Commerçants et assimilés } \\
\text { Employés civils, agents de service de la fonction publique } \\
\text { Policiers et militaires } \\
\text { Employés administratifs d'entreprise } \\
\text { Employés de commerce } \\
\text { Personnels de services directs aux particuliers } \\
\text { Retraités agriculteurs exploitants } \\
\text { Retraités artisans, commerçants ou chefs d'entreprise }\end{array}$ \\
\hline Défavorisée & $\begin{array}{l}\text { Ouvriers qualifiés } \\
\text { Ouvriers non qualifiés } \\
\text { Ouvriers agricoles } \\
\text { Retraités employés ou ouvriers } \\
\text { Chômeurs n'ayant jamais travaillé } \\
\text { Personnes sans activité professionnelle }\end{array}$ \\
\hline
\end{tabular}

Source : DEPP.

La distance entre séquences se calcule comme le nombre minimal d'opérations requises pour transformer une séquence en une autre, en utilisant des opérations d'insertion, de suppression ou de substitution d'états. Il existe différentes méthodes pour mesurer les distances entre séquences selon les opérations qu'elles utilisent. Le choix de la mesure relève d'un arbitrage temporel : les opérations d'insertion-suppression déforment la structure temporelle des séquences afin de faire émerger les enchaînements d'états communs composant les trajectoires, tandis que les opérations de substitution préservent la structure temporelle des séquences, afin de comparer les éléments à position constante (Lesnard \& De Saint Pol, op. cit. ; Robette 2011).

Dans cet article, nous utilisons la distance de Hamming dynamique (Lesnard, 2010). Cette mesure utilisant uniquement des opérations de substitution, nous privilégions les opérations qui permettent de rendre compte de la succession des étapes jalonnant les trajectoires des étudiants depuis leur entrée en L1. Nous mettons l'accent sur la temporalité des trajec- 
toires. Il s'agit ainsi de saisir à quel moment du parcours interviennent les éléments ne s’inscrivant pas dans le cadre d'une trajectoire universitaire linéaire (réorientation, changement d'établissement, sortie du système universitaire, etc.).

Les coûts sont estimés à partir des taux de transition entre états, selon la méthode décrite par Rohwer \& Pötter (2005). Ainsi, plus la probabilité de transition entre deux états est faible, plus le coût de substitution sera élevé (Robette \& Thibault, 2008). Une procédure de classification ${ }^{5}$ permet alors de construire une typologie des trajectoires étudiantes de chaque filière.

Nous utilisons ensuite deux modèles logit multinomiaux afin d'estimer les effets des variables socio-scolaires sur la probabilité d'appartenir à l'une ou l'autre des trajectoires types dans chaque filière ${ }^{6}$.

Nous menons une analyse multifacteur de dispersion de séquences ${ }^{7}$ (Studer $\&$ al., 2011) afin de statuer sur le poids de l'origine sociale, des performances scolaires des étudiants et de la spécialité choisie sur les trajectoires étudiantes.

Une enquête qualitative par entretiens semi-directifs a été menée entre 2012 et $2014^{8}$ auprès de 58 étudiants en droit et en AES, à Paris 1 et Paris 8, deux universités choisies pour leur proximité d'offre de formation (droit, sciences humaines) et pour leur ancrage distinct dans une métropole francilienne ségrégée (quartier Latin à Paris / Saint-Denis en SeineSaint-Denis). 18 des étudiants enquêtés étaient inscrits en M1 en 2012, les autres en L1 ont ensuite été enquêtés à nouveau en 2013, puis en 2014. Ce suivi qualitatif d'une quarantaine d'étudiants permet d'observer au plus près la construction de leur trajectoire universitaire, leurs aspirations et leurs ajustements, comme leurs mobilités entre établissements.

La trame d'entretien ${ }^{9}$ semi-directif (environ 1 h 30) reconstituait la trajectoire scolaire et résidentielle de chaque étudiant, le choix de l'université (après le bac, à l'entrée en Master), les mobilités et les transports utilisés, le rapport aux études et au lieu d'études (qui com-

5. Les typologies ont été déterminées en évaluant différents algorithmes de regroupement à l'aide de plusieurs mesures de qualité (standardisées). Ces évaluations sont reportées sur les figures A3 et A4, disponibles en annexes de la version électronique de l'article ; chaque sous-figure correspond à une évaluation des différents regroupements par une mesure de qualité. Nous avons décidé de retenir l'algorithme Ward qui produisait de bons résultats statistiques dans les deux cas et pour lequel les typologies faisaient sens au regard de notre problématique.

6. Les résultats sont disponibles à la demande auprès des auteurs. Dans la suite, les effets mentionnés sont significatifs au seuil de $5 \%$.

7. L'analyse de dispersion de séquences permet de mesurer l'association entre la variabilité des trajectoires individuelles et une variable explicative, en s'affranchissant de la simplification des données résultant d'une procédure de clustering. Dans le cadre d'une analyse multifacteur, l'apport additionnel de chaque co-variable est calculé lorsque les effets de l'ensemble des autres co-variables sont pris en compte.

8. Nous avons utilisé les données SISE les plus récentes disponibles au moment de l'enquête.

9. La trame complète figure en page 84 de la thèse de doctorat de Leïla Frouillou (2015) : https://halshs. archives-ouvertes.fr/tel-01274983. 
prend la sociabilité, les relations avec les professeurs, les méthodes de travail, etc.). Cette trajectoire était ensuite discutée à la fois par rapport à celle de la famille de l'enquêté et par rapport à celle de ses amis de lycée. Les résultats de l'enquête qualitative rendent également compte des circulations entre universités et secteurs extra-universitaires.

\subsection{Trois trajectoires-types des étudiants de droit et d'AES}

Les trajectoires des étudiants primo-entrants en L1 de droit et d'AES, dans une université d'Île-de-France, en 2007, sont retracées a posteriori durant cinq années ${ }^{10}$. Les états de ces trajectoires sont définis par la conjonction d'un niveau d'étude (licence et master), de l'établissement d'inscription (Paris et banlieue) et d'un secteur disciplinaire (droit ou AES et réorientation).

La distinction entre les universités parisiennes et de banlieue - résultant de contraintes techniques limitant le nombre d'états possibles - est peu satisfaisante au regard de l'hétérogénéité de ces deux groupes (Frouillou, 2015). Pour autant, elle rend compte d'une opposition centre-périphérie, que l'on peut lire dans les caractéristiques sociales et scolaires des publics, comme dans les discours des étudiants enquêtés. L'utilisation d'états relatifs, rendant compte des changements d'université ou de discipline par rapport à la situation initiale, permet d'identifier les trajectoires centripètes ou centrifuges à destination ou au départ de Paris et donc d'éventuels placements étudiants.

Les figures $\mathbf{A} \mathbf{1}$ et $\mathbf{A} \mathbf{2}$, en annexe de la version électronique de l'article, présentent les typologies ${ }^{11}$ de séquences pour le droit et l'AES. Pour chaque groupe, les trajectoires individuelles des étudiants sont représentées en ordonnée, avec en abscisse l’axe temporel. Les caractéristiques sociales et scolaires de ces étudiants sont détaillées dans les tableaux $\mathbf{1}$ et $\mathbf{2}$. Les résultats de l'analyse de dispersion de séquences sont présentés dans le tableau A1 en annexe de la version électronique de l'article.

10. Les données SISE comportant des anomalies avant le passage au LMD (licence-master-doctorat), nous les utilisons à partir de l'année universitaire 2007. Bien que les bases SISE permettent de suivre très précisément les trajectoires des étudiants, elles posent un important problème d'attrition. Ces trajectoires d'étudiants qui sortent de la base peuvent correspondre à un arrêt des études supérieures, mais aussi à une inscription dans un établissement d'enseignement supérieur ne relevant pas du champ SISE universités (écoles de management, d'ingénieurs, etc.) ou à une poursuite d'études dans un établissement d'enseignement supérieur à l'étranger. Les états correspondant à ces situations sont appelés " manquants ".

11. Nous avons agrégé les séquences identiques en suivant la méthode décrite dans Studer (2013). Les échantillons sur lesquels nous construisons les typologies contiennent les séquences représentatives, pondérées par leurs fréquences d'apparition. Les statistiques descriptives sont calculées sur les échantillons exhaustifs. 
Tableau 1. Caractéristiques socio-scolaires des étudiants inscrits pour la première fois en L1 de droit, en 2007, par trajectoires-types

\begin{tabular}{|c|c|c|c|c|c|c|c|c|c|c|}
\hline $\begin{array}{l}\text { Caractéristiques } \\
\text { socio- } \\
\text { scolaires }\end{array}$ & $\begin{array}{c}\text { Groupe } \\
1\end{array}$ & $\begin{array}{c}\text { Groupe } \\
2\end{array}$ & $\begin{array}{c}\text { Groupe } \\
3\end{array}$ & $\begin{array}{c}\text { Groupe } \\
4\end{array}$ & $\begin{array}{c}\text { Groupe } \\
5\end{array}$ & $\begin{array}{c}\text { Groupe } \\
6\end{array}$ & $\begin{array}{c}\text { Groupe } \\
7\end{array}$ & $\begin{array}{c}\text { Groupe } \\
8\end{array}$ & $\begin{array}{c}\text { Groupe } \\
9\end{array}$ & $\begin{array}{c}\text { Ensem- } \\
\text { ble }\end{array}$ \\
\hline \multicolumn{11}{|l|}{ PCS } \\
\hline Très favorisée & 39 & 54 & 55 & 50 & 37 & 38 & 59 & 69 & 64 & 51 \\
\hline Favorisée & 13 & 8 & 10 & 12 & 11 & 12 & 15 & 8 & 9 & 11 \\
\hline Moyenne & 25 & 23 & 23 & 21 & 23 & 20 & 17 & 17 & 19 & 21 \\
\hline Défavorisée & 23 & 15 & 13 & 17 & 29 & 31 & 8 & 6 & 9 & 17 \\
\hline \multicolumn{11}{|l|}{ Sexe } \\
\hline Féminin & 68 & 64 & 61 & 70 & 63 & 64 & 73 & 63 & 68 & 66 \\
\hline Masculin & 32 & 36 & 39 & 30 & 37 & 36 & 27 & 37 & 32 & 34 \\
\hline \multicolumn{11}{|l|}{ Nationalité } \\
\hline Française & 94 & 95 & 91 & 92 & 89 & 93 & 93 & 89 & 92 & 91 \\
\hline Étrangère & 6 & 5 & 9 & 8 & 11 & 7 & 7 & 11 & 8 & 9 \\
\hline \multicolumn{11}{|l|}{$\begin{array}{l}\text { Origine } \\
\text { géographique }\end{array}$} \\
\hline Essonne & 9 & 10 & 3 & 10 & 9 & 11 & 14 & 8 & 4 & 7 \\
\hline Hauts-de-Seine & 6 & 8 & 9 & 19 & 16 & 9 & 20 & 12 & 9 & 14 \\
\hline Paris & 16 & 23 & 42 & 8 & 9 & 4 & 25 & 34 & 50 & 25 \\
\hline Seine-et-Marne & 25 & 13 & 13 & 4 & 4 & 4 & 7 & 5 & 10 & 7 \\
\hline $\begin{array}{l}\text { Seine-Saint- } \\
\text { Denis }\end{array}$ & 12 & 8 & 4 & 9 & 13 & 14 & 7 & 8 & 5 & 8 \\
\hline Val-d'Oise & 13 & 13 & 1 & 12 & 16 & 14 & 3 & 12 & 2 & 9 \\
\hline Val-de-Marne & 7 & 8 & 5 & 13 & 10 & 15 & 8 & 3 & 5 & 9 \\
\hline Yvelines & 7 & 8 & 2 & 19 & 14 & 23 & 8 & 14 & 2 & 11 \\
\hline $\begin{array}{l}\text { Hors } \\
\text { Île-de-France }\end{array}$ & 6 & 10 & 20 & 6 & 9 & 5 & 7 & 5 & 14 & 11 \\
\hline \multicolumn{11}{|l|}{ Age } \\
\hline$<=18$ & 59 & 65 & 41 & 65 & 38 & 52 & 66 & 70 & 70 & 56 \\
\hline [19-20] & 35 & 34 & 39 & 30 & 45 & 44 & 34 & 27 & 27 & 34 \\
\hline$>=21$ & 16 & 1 & 20 & 5 & 17 & 4 & 0 & 3 & 3 & 10 \\
\hline \multicolumn{11}{|l|}{$\begin{array}{l}\text { Bourse sur } \\
\text { critères } \\
\text { sociaux }\end{array}$} \\
\hline Boursier & 28 & 26 & 11 & 23 & 26 & 28 & 10 & 17 & 16 & 20 \\
\hline Non boursier & 72 & 74 & 89 & 77 & 74 & 72 & 90 & 83 & 84 & 80 \\
\hline \multicolumn{11}{|l|}{ Série du bac } \\
\hline Littéraire & 23 & 23 & 25 & 23 & 19 & 30 & 27 & 15 & 22 & 22 \\
\hline Économique & 50 & 46 & 41 & 52 & 33 & 46 & 54 & 52 & 49 & 45 \\
\hline Scientifique & 11 & 13 & 12 & 14 & 7 & 7 & 12 & 22 & 21 & 14 \\
\hline Technologique & 13 & 16 & 13 & 9 & 27 & 15 & 7 & 6 & 3 & 12 \\
\hline $\begin{array}{l}\text { Professionnel ou } \\
\text { dispensé }\end{array}$ & 3 & 3 & 9 & 4 & 14 & 3 & 0 & 5 & 4 & 6 \\
\hline
\end{tabular}


Lecture : dans le groupe 1 («Après un an, réorientation dans une autre université de banlieue »), $39 \%$ des étudiants ont une origine sociale très favorisée. On distingue trois ensembles de types de trajectoires : interuniversitaires (en gris foncé dans les tableaux), même université (en blanc), ou sorties du système universitaire (en grisé).

Champ : 7718 trajectoires d'étudiants inscrits pour la première fois en L1 de droit dans une université francilienne en 2007.

Note : Groupe 1 ( $2 \%$ des trajectoires) : Après un an, réorientation dans une autre université de banlieue ; Groupe $2(0,5 \%)$ : Après un an, inscription dans une autre université de banlieue ; Groupe $3(18 \%)$ : Sortie du système Paris ; Groupe 4 (32\%) : Droit dans la même université en banlieue ; Groupe 5 $(21 \%)$ : Sortie du système banlieue ; Groupe $6(2 \%)$ : Réorientation dans la même université en banlieue ; Groupe $7(0,7 \%)$ : Après un an, réorientation dans une autre université parisienne ; Groupe $8(0,8 \%)$ : Inscription en droit dans une autre université parisienne ; Groupe $9(23 \%)$ : Droit dans la même université à Paris.

Source : « SISE universités inscriptions » 2007-2011, « SISE universités résultats » 2007-2010 et « AGLAE » 2007-2011.

Tableau 2. Caractéristiques socio-scolaires des étudiants inscrits pour la première fois en L1 AES, en 2007, par trajectoires-types

\begin{tabular}{|c|c|c|c|c|c|c|c|c|c|}
\hline $\begin{array}{c}\text { Caractéristiques } \\
\text { socio- } \\
\text { scolaires }\end{array}$ & $\begin{array}{c}\text { Groupe } \\
1\end{array}$ & $\begin{array}{c}\text { Groupe } \\
2\end{array}$ & $\begin{array}{c}\text { Groupe } \\
3\end{array}$ & $\begin{array}{c}\text { Groupe } \\
4\end{array}$ & $\begin{array}{c}\text { Groupe } \\
5\end{array}$ & $\begin{array}{c}\text { Groupe } \\
6\end{array}$ & $\begin{array}{c}\text { Groupe } \\
7\end{array}$ & $\begin{array}{c}\text { Groupe } \\
8\end{array}$ & Ensemble \\
\hline \multicolumn{10}{|l|}{ PCS } \\
\hline Très favorisée & 31 & 23 & 25 & 22 & 26 & 37 & 33 & 35 & 27 \\
\hline Favorisée & 16 & 6 & 12 & 14 & 10 & 10 & 12 & 17 & 12 \\
\hline Moyenne & 23 & 27 & 23 & 28 & 26 & 29 & 27 & 31 & 25 \\
\hline Défavorisée & 30 & 44 & 41 & 36 & 38 & 24 & 28 & 17 & 36 \\
\hline \multicolumn{10}{|l|}{ Sexe } \\
\hline Féminin & 74 & 63 & 66 & 65 & 52 & 55 & 71 & 61 & 65 \\
\hline Masculin & 26 & 37 & 34 & 35 & 48 & 45 & 29 & 39 & 35 \\
\hline \multicolumn{10}{|l|}{ Nationalité } \\
\hline Française & 82 & 90 & 89 & 85 & 88 & 87 & 79 & 74 & 86 \\
\hline Étrangère & 18 & 10 & 11 & 15 & 12 & 13 & 21 & 26 & 14 \\
\hline \multicolumn{10}{|l|}{$\begin{array}{l}\text { Origine } \\
\text { géographique }\end{array}$} \\
\hline Essonne & 13 & 10 & 16 & 9 & 10 & 3 & 7 & 4 & 12 \\
\hline Hauts-de-Seine & 10 & 8 & 11 & 8 & 9 & 10 & 14 & 13 & 11 \\
\hline Paris & 7 & 8 & 7 & 10 & 7 & 39 & 32 & 31 & 14 \\
\hline Seine-et-Marne & 12 & 17 & 8 & 10 & 9 & 11 & 10 & 11 & 9 \\
\hline $\begin{array}{l}\text { Seine-Saint- } \\
\text { Denis }\end{array}$ & 19 & 23 & 18 & 22 & 20 & 11 & 7 & 13 & 17 \\
\hline Val-d'0ise & 14 & 15 & 8 & 7 & 7 & 2 & 3 & 0 & 7 \\
\hline Val de Marne & 13 & 4 & 12 & 22 & 16 & 16 & 18 & 6 & 15 \\
\hline Yvelines & 8 & 10 & 13 & 8 & 17 & 1 & 1 & 4 & 10 \\
\hline $\begin{array}{l}\text { Hors } \\
\text { Île-de-France }\end{array}$ & 4 & 6 & 6 & 4 & 4 & 7 & 7 & 19 & 6 \\
\hline \multicolumn{10}{|l|}{ Age } \\
\hline$<=18$ & 51 & 62 & 26 & 39 & 42 & 38 & 56 & 54 & 36 \\
\hline [19-20] & 40 & 31 & 51 & 44 & 48 & 50 & 38 & 37 & 46 \\
\hline$>=21$ & 9 & 7 & 23 & 17 & 10 & 12 & 6 & 9 & 18 \\
\hline
\end{tabular}




\begin{tabular}{|l|c|c|c|c|c|c|c|c|c|}
\hline $\begin{array}{l}\text { Bourse sur } \\
\text { critères } \\
\text { sociaux }\end{array}$ & & & & & & & & & \\
Boursier & 33 & 52 & 35 & 43 & 46 & 31 & 34 & 33 & 36 \\
Non boursier & 67 & 48 & 65 & 57 & 54 & 69 & 66 & 67 & 64 \\
\hline Série du bac & & & & & & & & & \\
Littéraire & 6 & 6 & 5 & 6 & 9 & 3 & 5 & 6 & 5 \\
Économique & 57 & 65 & 29 & 55 & 38 & 46 & 66 & 57 & 42 \\
Scientifique & 8 & 6 & 3 & 4 & 6 & 5 & 6 & 24 & 5 \\
Technologique & 17 & 21 & 39 & 22 & 46 & 33 & 14 & 7 & 31 \\
Professionnel ou & 11 & 2 & 24 & 14 & 1 & 13 & 9 & 6 & 18 \\
dispensé & 11 & 24 & & \\
\hline
\end{tabular}

Lecture : dans le groupe 1 (« Réorientations principalement en banlieue »), $31 \%$ des étudiants ont une origine sociale très favorisée.

Champ : 2218 trajectoires d'étudiants inscrits pour la première fois en L1 AES dans une université francilienne en 2007.

On distingue trois ensembles de types de trajectoires : interuniversitaires (en gris foncé dans les tableaux), même université (en blanc), ou sorties du système universitaire (en grisé).

Note : Groupe $1(5 \%)$ : Réorientations principalement en banlieue ; Groupe $2(2 \%)$ : Réorientations après un an dans une autre université en banlieue ; Groupe $3(51 \%)$ : Sorties du système universitaire banlieue ; Groupe 4 (16\%) : AES dans la même université en banlieue ; Groupe 5 (3\%) : Interruptions en banlieue et reprises d'études ; Groupe 6 (11\%) : Sorties du système Paris ; Groupe 7 (9\%) : AES dans la même université à Paris ; Groupe 8 (2 \%) : Réorientations dans la même université à Paris.

Source : « SISE universités inscriptions » 2007-2011, « SISE universités résultats » 2007-2010 et « AGLAE » 2007-2011.

Ainsi, on distingue trois ensembles de types de trajectoires : interuniversitaires (en gris foncé dans les tableaux), même université (en blanc), ou sorties du système universitaire (en grisé). Il s'agit maintenant d'interroger ces types au regard de nos deux hypothèses introductives.

\section{Les trajectoires majoritaires au prisme 3 des mécanismes de la sélection sociale et scolaire}

Nous détaillons ici les trajectoires les plus fréquentes des étudiants franciliens en droit et AES : d'abord celles des étudiants qui restent dans la même université, puis celles qui correspondent à une sortie du système universitaire.

\subsection{Les trajectoires étudiantes au sein d'une même université}

Les trajectoires restreintes à un seul établissement concernent près de $57 \%$ des étudiants en droit entrant pour la première fois en L1 en Île-de-France, en 2007, contre $28 \%$ en AES.

Les tableaux 1 et $\mathbf{2}$ illustrent les disparités entre Paris et sa banlieue et entre filières. Quelle que soit la typologie, les groupes de trajectoires d'étudiants inscrits à Paris (groupe 9 pour le droit et 7 pour l'AES) sont composés d'étudiants socialement et scolairement plus favo- 
risés que ceux des trajectoires d'étudiants inscrits en banlieue (groupes 4 et 6 pour le droit et 4 pour l'AES).

En droit, les étudiants du groupe 9 sont à $64 \%$ issus d'un milieu très favorisé, contre respectivement $50 \%$ et $38 \%$ dans les groupes 4 et 6 . Ils sont titulaires d'un bac scientifique à $21 \%$, contre $14 \%$ et $7 \%$ dans les groupes 4 et 6 . Ces chiffres sont corroborés par le fait qu'en droit, la probabilité de suivre toutes ses études dans la même université parisienne (groupe 9) plutôt que dans la même université de banlieue (groupe 4) est moins élevée pour les étudiants d'origine sociale défavorisée et pour les titulaires d'un bac technologique, alors qu'elle est plus élevée pour les étudiants titulaires d'un bac scientifique et pour les élèves les plus jeunes.

En AES, les étudiants du groupe 7 sont issus d'un milieu très favorisé à $33 \%$, contre $22 \%$ dans le groupe $4.56 \%$ ont 18 ans ou moins à l'entrée en L1, contre $39 \%$ dans le groupe 4, et sont titulaires d'un bac général à $77 \%$, contre $65 \%$ dans le groupe 4 .

Ces trajectoires restreintes à une même université comportent aussi des réorientations disciplinaires qui ne correspondent pas aux mêmes étudiants en droit et en AES. Cela traduit la position distincte qu'occupent ces filières dans la hiérarchie disciplinaire.

Les étudiants inscrits en droit dans une université de banlieue ${ }^{12}$ et qui se réorientent dans la même université (groupe 6) sont moins favorisés socialement et scolairement que ceux qui poursuivent leurs études en droit dans la même université de banlieue (groupe 4). Par rapport aux étudiants qui ont suivi toutes leurs études dans la même université de banlieue, la probabilité de se réorienter dans la même université de banlieue augmente pour les étudiants qui ont une origine sociale défavorisée et qui sont titulaires d'un bac technologique.

L'enquête qualitative révèle que les facteurs qui jouent un rôle dans la poursuite d'études au sein du même établissement sont la familiarité avec le lieu d'études, avec les enseignants et les étudiants, mais aussi les facilités administratives de réinscription dans le même établissement ${ }^{13}$.

12. Nous ne mentionnons pas le cas des étudiants de droit inscrits dans une université parisienne qui se réorientent, car ils s'inscrivent en majorité dans une autre université, appartenant donc à un groupe de trajectoires interuniversitaires (groupe 7).

13. L'argument de la "proximité " avec le lieu d'études est mobilisé en entretien par les étudiants pour expliquer le choix universitaire en L1 : il masque souvent d'autres motifs (réputation, conseils familiaux et amicaux) et est à géométrie très variable au cours de la trajectoire d'études (selon le mode de transport utilisé, les autres lieux de travail ou de loisirs, les éventuels déménagements). La " proximité " n’est plus évoquée comme motif pour expliquer le choix universitaire en master, au profit d'enjeux institutionnels (réputation, spécialisation de la formation, modalités d'inscription, liens avec les professeurs, etc.). Les trajets constituent alors des éléments secondaires dans la justification du choix de l'université, comme l'illustre plus loin une citation de Clémence. 
Émilie (droit, M1, Paris 8, père chef d'entreprise et mère assistante sociale, habitant dans le 93, bachelière L) évoque les liens tissés avec les enseignants et les difficultés administratives liées à un éventuel changement d'université :

"Après, j'ai l'avantage aussi d'avoir fait tout mon cursus dans la même université, donc je connais les professeurs, je sais un peu ce qu'ils attendent. [...] Moi ça m’a pas posé de problème, mais je sais que j'ai des amis qui n’ont pas réussi à partir parce quion leur a donné leurs notes d'examen en retard."

Ces éléments se retrouvent également à Paris 1, comme l'illustre le cas de Marc (AES, L3, Paris 1, père informaticien et mère enseignante, habitant à Paris, bachelier ES) :

"Paris 1, je m'y sens vraiment bien. Et si j'ai aussi envie de rester, c'est aussi parce qu'il y a autour... Le volley, la fac, l'association d'AES."

Ces entretiens permettent de comprendre pourquoi la plupart des trajectoires universitaires sont limitées à un seul établissement, ce type de poursuite d'études permettant de s'appuyer sur le réseau social étudiant, enseignant et administratif développé au cours du temps par les étudiants. Cela permet de favoriser les processus d'affiliation et l'apprentissage du métier d'étudiant (Coulon, 1997).

\subsection{Des trajectoires de sortie qui varient en fonction de la hiérarchie universitaire}

Pour chaque typologie, la part des trajectoires de sortie est d'autant plus élevée que celle des trajectoires au sein d'une même université est faible, selon un fonctionnement de vases communicants. L'analyse menée par la DEPP (op. cit., p. 200) précise que ces taux de sortie des premiers entrants à l'université sont particulièrement élevés en AES (39\%).

Les tableaux 1 et $\mathbf{2}$ révèlent que ces trajectoires sont principalement le fait d'étudiants socialement et scolairement défavorisés.

Les étudiants inscrits en droit à Paris, sortant du système universitaire (groupe 3), sont moins souvent issus d'une catégorie sociale très favorisée que ceux qui poursuivent leurs études de droit dans la même université parisienne (groupe 9) (55\%, contre 64\%); ils sont moins nombreux à avoir obtenu leur bac à 18 ans ou avant $(41 \%$, contre $70 \%)$ et à être titulaire d'un bac général (78\%, contre $92 \%)$.

En droit, la probabilité de sortir du système universitaire après une première inscription en banlieue (groupe 5) plutôt que de continuer ses études dans la même université de banlieue (groupe 4) augmente pour les étudiants qui ont une origine sociale moyenne ou défavorisée, pour les garçons, pour les plus âgés et pour ceux qui sont titulaires d'un bac technologique ou professionnel ou qui en ont été dispensés (le même effet est constaté en AES). Cette probabilité diminue pour les étudiants étrangers et pour ceux qui ont un bac scientifique. 
La série du bac et l'âge d'obtention de ce diplôme sont deux variables clés pour saisir l'échec dans les premières années universitaires (Beaupère \& Boudesseul, op. cit.). On peut donc penser qu'une part importante des étudiants sortant du système se trouve en situation d'échec.

Cette élimination des étudiants de classe populaire et/ou des bacheliers technologiques et professionnels tient notamment à l'inadéquation entre les attentes professorales souvent implicites ${ }^{14}$ (Felouzis, 1997) et ce qu'en comprennent des étudiants peu familiers de la culture scolaire et universitaire. Les cours magistraux, ainsi que le manque d'intégration sociale en première année constituent des éléments défavorables à la réussite des étudiants peu assurés scolairement (Beaud, 2008).

Cependant, les processus de sélections sociale et scolaire ne sont pas les mêmes dans les deux disciplines, et témoignent de la place qu'elles occupent dans la hiérarchie universitaire.

En droit, les sorties du système universitaire concernent davantage les étudiants inscrits dans une université parisienne ( $43 \%$, contre $37 \%$ pour ceux inscrits dans une université de banlieue). La sur-sélection des universités parisiennes en droit n'apparaît pas en AES, où les sorties du système universitaire concernent davantage les universités de banlieue (67\%) que les universités parisiennes (46\%).

Cela rappelle les résultats de Nicourd \& al., (2013) quant à une certaine homogénéisation scolaire des publics entre Paris 13 et Versailles-Saint-Quentin. Les sortants sont moins dotés scolairement que ceux qui restent, mais ils ne sont pas plus défavorisés socialement. Cette disjonction entre caractéristiques sociales et scolaires pourrait tenir à l'hétérogénéité des trajectoires de sortie. L'AES constituant pour beaucoup d'étudiants un choix "par défaut ", le fait de ne pas se réinscrire n'est pas forcément synonyme d'échec, certains étudiants poursuivant leurs études dans d'autres champs de l'enseignement supérieur et d'autres s'insérant sur le marché du travail.

Bien que de nombreuses sorties correspondent aux caractéristiques sociales et scolaires mises en évidence dans les travaux sur le décrochage, l'enquête qualitative souligne l'importance des réorientations et des longues interruptions d'études.

Ainsi, après un passage par l'université, Julia (droit, L1, Paris 1, père avocat, mère vendeuse dans le commerce du luxe, habitant Paris, bachelière L) est partie un an à Taïwan avant de s'inscrire en Bachelor à Copenhague ; Nimal (droit, L1, Paris 1, père employé

14. Ces implicites renvoient à l'apprentissage du "métier d'étudiant " (Coulon, 1997), processus d'affiliation qui consiste à s'approprier les attentes des enseignants et à organiser son travail pour y répondre (gérer son temps, savoir solliciter les enseignants pour des conseils méthodologiques ou préciser des consignes, être attentif à la syntaxe et l'orthographe, savoir organiser un propos en plusieurs parties, se référer à des manuels, etc.), ce qui est souvent considéré comme l'acquisition d'une autonomie dans le travail personnel universitaire. 
dans un supermarché et mère employée municipale, habitant dans le 92, bachelier ES) est entré dans un IEP (Institut d'Études Politiques) ; et Catherine (AES, L1, Paris 1, père ouvrier et mère au foyer, habitant également dans le 92 , bachelière ES) est entrée en classe préparatoire littéraire.

L'hétérogénéité de ces trajectoires renvoie ainsi non seulement à la reproduction des positions sociales par l'université, mais aussi à des circulations : "En recouvrant sous un même terme ('décrochage', 'abandon', 'échec', etc.) des réalités extrêmement diverses, on se condamne à ne lire les parcours étudiants qui ont le tort de ne pas être parfaitement linéaires qu'en négatif [...]. [Les premiers cycles] constituent un espace tampon, de régulation des flux successifs de bacheliers qui agit comme un lieu à la fois d'expérimentation et de construction progressive de parcours intellectuels véritables et de maintien des hiérarchies sociales et scolaires. " (Bodin, Orange, 2013 p. 111). Ces circulations peuvent également s'observer entre établissements.

\section{Des trajectoires interuniversitaires minoritaires, mais révélatrices de placements}

L'un des principaux résultats des typologies réalisées est de révéler la très faible part qu'occupent les trajectoires interuniversitaires franciliennes ( $4 \%$ en droit et $5 \%$ en AES) par rapport aux autres types de trajectoires. Mais ces typologies conduisent à sous-estimer cette proportion dans la mesure où une partie de ces trajectoires interuniversitaires est incluse dans d'autres groupes.

En droit, dans le groupe 6 "Réorientations dans le même établissement de banlieue " (2\% de l'ensemble des trajectoires), un tiers des étudiants de banlieue qui ont changé de discipline en licence finissent par changer d'établissement (en banlieue) pour s'inscrire en master de droit. Dans le groupe 4 "Droit dans la même université en banlieue " (32\%), certaines trajectoires sont des trajectoires interuniversitaires.

Lorsque le changement d'université arrive après la licence, ces trajectoires sont intégrées dans les groupes 9 (s'ils continuent leurs études dans la même université parisienne) et 4 (s'ils le font en banlieue). Baron \& al. (op. cit.) montrent que $10 \%$ des trajectoires entre le premier et le deuxième cycle correspondent à un changement d'établissement ${ }^{15}$.

Ces constats tranchent avec les résultats de l'enquête qualitative, où la surreprésentation des trajectoires interuniversitaires est due, d'une part, au fait que certains types de trajectoires interuniversitaires ne sont pas inclus dans notre approche (par exemple les tra-

15. Notre approche quantitative met en évidence des résultats similaires puisque, pour les étudiants inscrits en AES et en droit qui ne quittent pas le système universitaire (50\% des trajectoires), les mobilités représentent $5 \%$ des trajectoires. 
jectoires depuis ou à destination de la province) et, d'autre part, au fait que l'approche qualitative comporte nécessairement un biais de sélection. Chercher des étudiants volontaires pour évoquer leur parcours scolaire implique une certaine surreprésentation des étudiants estimant leur parcours suffisamment " original " (réorientation, changement d'établissement, reprise d'études, etc.) pour être raconté.

Ces trajectoires participent-elles à une attraction des étudiants les mieux dotés scolairement et socialement vers les établissements parisiens?

En droit ${ }^{16}$, les trajectoires interuniversitaires ${ }^{17}$ des étudiants sont de deux types : de la banlieue vers Paris (groupe 8) ou l'inverse (groupe 2).

Le tableau 1 montre que les étudiants qui poursuivent leurs études en droit dans une université parisienne, après avoir été inscrits majoritairement dans une université de banlieue (groupe 8), ont un profil social et scolaire très proche des étudiants qui continuent en droit dans leur université parisienne d'origine (groupe 9). Ils sont ainsi $69 \%$ et $64 \%$ à être issus d'un milieu social très favorisé, $70 \%$ et $70 \%$ à avoir 18 ans ou moins au moment de leur première inscription en L1, $17 \%$ et $16 \%$ à percevoir une bourse sur critères sociaux, $52 \%$ et $49 \%$ à être titulaires d'un bac économique, et $22 \%$ et $21 \%$ d'un bac scientifique.

Les étudiants qui ont commencé leurs études en banlieue avant de rejoindre une université parisienne ont des caractéristiques sociales différentes de ceux qui n'ont pas quitté leur université de banlieue d'origine.

Les étudiants qui poursuivent en droit dans une autre université, après avoir été inscrits majoritairement dans une université parisienne (groupe 2), ont des caractéristiques proches de ceux qui continuent en droit dans leur université de banlieue d'origine (groupe 4). Ils sont ainsi $54 \%$ et $50 \%$ à avoir une origine très favorisée, $65 \%$ et $65 \%$ à avoir 18 ans ou moins au moment de leur première inscription et $26 \%$ et $23 \%$ à percevoir une bourse.

Ces résultats montrent qu'un ajustement social et scolaire s'opère à travers les trajectoires interuniversitaires. L'enquête qualitative permet de comprendre les motivations de ces étudiants mobiles et le choix de l'université d'arrivée.

Pour expliquer leur changement d'établissement au cours de leur trajectoire, Imran (AES, M1, Paris 1, mère femme au foyer, habitant dans le 93, bachelier ES ; passage de Paris 8 à Paris 1 en L3) et Clémence (droit, M2, Paris 1, père représentant et mère assistante familiale, habitant dans le 95 , bachelière $S$; passage de Cergy à Paris 1 en M1),

16. D'un point de vue quantitatif, les trajectoires interuniversitaires en AES sont difficilement isolables, car elles sont entremêlées à des trajectoires de réorientations (groupes 1 et 2) et d'interruptions et de reprises d'études (groupe 5).

17. Sont exclues les trajectoires interuniversitaires comprenant des réorientations (groupes 1 et 7), leurs implications en termes de différenciations de publics dépassant le cadre de cet article. 
soulignent le prestige de Paris 1, mais insistent aussi sur d'autres éléments, tels que le temps de trajet et la vie étudiante parisienne pour Clémence, par exemple :

"En fait, [le master de] Nanterre me plaisait plus, mais pour être à la Sorbonne, j’ai pris celui de la Sorbonne. [...] Je me suis dit que c'était bien pour la réputation, parce que justement, après être partie à l'étranger, tu te rends compte que la Sorbonne, les étrangers, ils ne connaissent que ça. [...] Aussi, il y avait des questions de trajet. C'est moins long de chez moi que d'aller à Nanterre."

$\mathrm{L}:$ : Et à Cergy par contre, il n'y avait pas le master que tu voulais ? C'est pour ça que tu es partie et/ou c'est parce que tu voulais changer de fac?"

"Bah je voulais changer, je ne voulais plus rester à Cergy, c'est une bonne ambiance et tout... Mais c'est un peu isolé des autres étudiants, parce que tous nos amis sont à Paris. Et nous on a une vie étudiante à Cergy, mais c'est complètement différent que quand tu es à Paris. "

La vie parisienne étudiante occupe aussi une place centrale dans les trajectoires de Florence (droit, M1, Paris 1, parents professeurs des universités en médecine, habitant Tours, puis Paris, bachelière $S$; passage de Tours à Paris en L3) :

"Paris 1, c’était un peu le fantasme de Province. Enfin, Paris 1 et Assas. Je ne voulais pas quitter Tours si ce n'était pas pour intégrer une fac un peu..."

et de Shaïnez (droit, L3, Paris 1, père cadre dans l'industrie et mère cadre supérieure, habitant Alger, puis Paris, bachelière $S$; réorientation de L1 de médecine à Lyon à L1 droit à Paris 1) :

"Mes parents voulaient que je vienne à Paris. [...] Le choix se portait entre Paris 1 et Paris 2. Et finalement, ceetait Paris 1. Parce qu'en fait, déjà, quand on regarde les classements des facultés de droit, ce sont les deux meilleures."

Ces étudiantes soulignent à la fois le prestige international de Paris 1 et la place des universités parisiennes dans leur réseau social et familial.

À l'inverse, certains étudiants décident de quitter la région, notamment à cause d'une sélectivité de plus en plus forte avec l'avancée dans les niveaux d'études. Le cas de Tatiana, notamment (AES, L2, Rennes, père directeur informatique et mère professeur des écoles, habitant Paris, bachelière ES), montre que cet enjeu de la sélection peut aussi se mêler aux contraintes familiales (décohabitation, conflits, etc.).

Si Mélanie (AES, L3, Paris 12, père cadre et mère assistante de gestion, habitant dans le 93, bachelière ES ; passage de Paris 8 à Paris 12 en M1) change d'université en raison d'une spécialisation de l'offre de formation (master banques et marchés financiers de Paris 12), les mobilités interuniversitaires de Maxime (droit, M1, Paris 10, mère cadre, habitant dans le 95, bachelier S; passage de Paris 8 à Paris 10 en M1) et de Victoire (droit, M1, Paris 10, père chauffeur de taxi et mère ouvrière, habitant dans le 93 , bachelière L; passage de Paris 8 à Paris 10 en M1) sont guidées par une volonté de valider un master dans une 
université plus prestigieuse que Paris 8 . Ces deux étudiants souhaitaient entrer à Paris 1 en master et ont obtenu leur deuxième ou troisième choix : Paris 10 Nanterre. Ces deux trajectoires, révélatrices de stratégies universitaires similaires, se sont construites selon une temporalité différente puisque, contrairement à Victoire qui avait vainement postulé en L1 à Paris 1, Maxime n'avait pas souhaité entrer dans cette université après son bac, obtenu de justesse. Son inscription à Paris 10 en M1 découle d'une certaine réassurance scolaire construite tout au long de sa licence à Paris 8.

Les trajectoires interuniversitaires font jouer à la fois le capital scolaire des étudiants (notamment à travers la sélection de leur dossier par l'établissement) et leurs capitaux économiques (possibilité de déménager) et sociaux (liens familiaux et amicaux, voire amoureux).

Les analyses de séquences révèlent que les étudiants les mieux dotés scolairement et socialement sont ceux qui connaissent des trajectoires interuniversitaires vers Paris (et inversement), ce que confirme l'enquête. Cette dynamique ne se résume cependant pas à une attraction des étudiants les mieux dotés vers les universités parisiennes. Certaines trajectoires interuniversitaires s'inscrivent en effet dans le schéma aréolaire (Paris vers Paris ou banlieue vers banlieue) témoignant des circulations et complémentarités entre universités parisiennes ou de banlieue.

Les trajectoires interuniversitaires entre établissements de banlieue soulignent les fines distinctions qu'opèrent les étudiants entre ces universités, Paris 10 Nanterre en droit étant plutôt associée aux universités parisiennes. De manière plus générale, le suivi qualitatif des étudiants révèle que leur connaissance du paysage universitaire, francilien comme national, s'étoffe au fil du temps. Cela traduit une certaine construction de capital scolaire en cours d'études, permettant de développer de nouvelles stratégies faisant jouer, notamment, des mobilités.

\section{Conclusion}

L'objectif de cet article était d'observer les différenciations de publics entre universités franciliennes, en considérant les trajectoires d'étudiants inscrits en droit et AES, au regard de deux processus : la sélection sociale des étudiants (trajectoires de sortie du système universitaire, différenciées selon les établissements et les disciplines) et l'attraction des étudiants les mieux dotés envers les établissements les mieux placés dans la hiérarchie symbolique universitaire (trajectoires interuniversitaires).

L'originalité tient ici au croisement d'une approche par les trajectoires étudiantes (à la fois qualitative et quantitative) et d'une échelle d'analyse régionale, à partir de la comparaison de deux disciplines universitaires.

Nos résultats confirment ceux de Nicourd \& al. (2013, p. 72) concernant l'existence d'un " processus d'homogénéisation scolaire [qui] s'opère par l'élimination des étudiants à 
faible capital scolaire ", mais aussi par les trajectoires interuniversitaires des étudiants les mieux dotés.

L'utilisation des méthodes d'analyse de séquences nous a permis de construire une typologie exhaustive des trajectoires étudiantes franciliennes en droit et en AES, qualifiant les ordres de grandeur de quatre types de trajectoires universitaires.

On constate d'abord la prédominance des trajectoires stables (même établissement ou même catégorie d'établissements), confirmant les résultats de Baron \& al. (2007). Un deuxième type de trajectoires, celles de sortie du système universitaire, se différencie en fonction de la place occupée par les filières dans la hiérarchie disciplinaire : la sélectivité est plus forte dans les universités parisiennes en droit, quand en AES, ce sont les universités de banlieue qui sont plus sélectives (homogénéisation des publics). Les trajectoires interuniversitaires, dont l'analyse de séquences souligne la place minoritaire, démontrent un réajustement social et scolaire des publics étudiants entre universités.

L'utilisation d'un matériau qualitatif permet ici d'expliciter les facteurs de mobilité entre universités et surtout de souligner la diversité de ces trajectoires interuniversitaires. Elles traduisent la finesse des hiérarchies universitaires franciliennes, changeantes selon la discipline et le niveau d'études.

Enfin, l'analyse de dispersion de séquences montre, d'une part, que la discipline différencie davantage les trajectoires des étudiants que l'origine sociale ; d'autre part, que les performances scolaires (appréhendées à partir de la série du bac) ont un effet de la même intensité que la discipline sur la variabilité des trajectoires. Ces résultats confirment la pertinence de la comparaison entre le droit et l'AES : les disciplines et filières correspondent à des placements distincts dans l'espace de l'enseignement supérieur.

Au-delà des incontournables hiérarchies disciplinaires, il existe également des spécificités tenant aux établissements, et qui varient selon la discipline, sa sélectivité et sa répartition entre les établissements.

En conclusion, l'analyse de séquences comme l'enquête auprès des étudiants ont permis de révéler l'existence d'un dernier type de trajectoires minoritaires en AES : les trajectoires d'expérimentation, qui se construisent autour de plusieurs interruptions, reprises d'études, changements d'établissements et/ou de filières. Leur analyse constitue un prolongement de ce travail, susceptible de révéler toute l'importance des temporalités dans la construction des trajectoires étudiantes, ces dernières ne pouvant se comprendre qu'au regard de trajectoires sociales (événements familiaux, parcours scolaire antérieur, vie professionnelle, etc.). 


\section{Bibliographie}

Baron M., Berroir S., Cattan N., Lesecq G., Saint-Julien T. (2007), « Des Universités en concurrence ", in Saint-Julien T., Le Goix R. (dir.), La métropole parisienne : centralités, inégalités, proximités, Paris, Belin, pp. 65-88.

Beaud S. (2008), "Enseignement supérieur : la 'démocratisation scolaire' en panne ", Formation Emploi, $\mathrm{n}^{\circ} 101$, pp. 149-165.

Beaupère N., Boudesseul G. (2009), Sortir sans diplôme de l'Université : comprendre les parcours d'étudiants "décrocheurs ", Paris, La Documentation française, 221 p.

De Berny C. (2008), « Les étudiants franciliens inscrits en université : origine sociale, cursus et modes de vie ", Etude de l'IAU, 40 p.

Berroir S., Cattan N., Saint-Julien T. (2005), « L'attraction des universités des villes nouvelles franciliennes ", Les cahiers de l'IAURIF, n 143, pp. 76-84.

Borras I., Épiphane D., Lemistre P., Ryk G. (2012), « Étudier en Licence : parcours et insertion ", Relief Céreq n³6, 240 p.

Bodin R., Orange S. (2013), L'université n'est pas en crise : les transformations de l'enseignement supérieur, Bellecombe-en-Bauges, Éditions du Croquant, 216 p.

Bodin R., Millet M. (2011), "L'université, un espace de régulation. L'abandon' dans les lers cycles à l'aune de la socialisation universitaire ", Sociologie, vol. 2, n 3, pp. 225-242.

Bourdieu P. (1994), Raisons pratiques : sur la théorie de l'action, Paris, Éditions du Seuil, $24 \mathrm{p}$.

Bourdieu P., Passeron J.-C. (1964), Les Héritiers. Les étudiants et la culture, Paris, Les Editions de Minuit, 192 p.

Cordazzo P. (2014), Parcours étudiants : de la formation à l'insertion professionnelle, Habilitation à Diriger les Recherches, Université Montesquieu-Bordeaux IV, 199 p.

Coulon A. (1997), Le métier d'étudiant : l'entrée dans la vie universitaire, Paris, PUF, 219 p.

DEPP (2015), Repères et références statistiques, 423 p.

Duru-Bellat M. (2002), Les inégalités sociales à l'école : genèse et mythes, Paris, PUF, 250 p.

Erlich V., Verley E. (2010), "Une relecture sociologique des parcours des étudiants français : entre segmentation et professionnalisation ", Éducation et Sociétés, n 26, pp. 71-88.

Felouzis G. (1997), "Les étudiants et la sélection universitaire ", Revue française de pédagogie, $\mathrm{n}^{\circ} 119(1)$, pp. 91-106.

Frouillou L. (2015), Les mécanismes d'une ségrégation universitaire francilienne : carte universitaire et sens du placement étudiant, thèse de doctorat, Université Paris 1 Panthéon Sorbonne, $522 \mathrm{p}$. 
Girault J., Lescure J.-C., Vadelorge L. (2012), « Paris XIII : histoire d'une université en banlieue (1970-2010)», Paris, Berg International éditeurs, 303 p.

Hugrée C. (2010), L'échappée belle : parcours scolaires et cheminements professionnels des étudiants d'origine populaire diplômés de l'Université (1970-2010), thèse de doctorat, Université de Nantes, 498 p.

Lefeuvre S. (2003), « La filière AES, un renouvellement de la professionnalisation et de la démocratisation dans l'Université française ?", Cahiers de la recherche sur l'éducation et les savoirs, $\mathrm{n}^{\circ} 3$, pp. 111-128.

Lesnard L. (2010), "Setting Cost in Optimal Matching to Uncover Contemporaneous Socio-Temporal Patterns”, Sociological Methods \& Research, n 38(3), pp. 389-419.

Lesnard L., de Saint Pol T. (2006), "Introduction aux méthodes d'appariement optimal ", Bulletin de méthodologie sociologique, $\mathrm{n}^{\circ}$ 90, pp. 5-25.

Merle P. (2013), «La catégorie socio-professionnelles des parents dans les fiches administratives des élèves ", Socio-logos, $\mathrm{n}^{\circ} 8$.

Nicourd S., Samuel O., Vilter S. (2011), «Les inégalités territoriales à l'université : effets sur les parcours des étudiants d'origine populaire ", Revue française de pédagogie, n¹76(3), pp. 27-40.

Nicourd S., Samuel O., Vilter S. (2013), «L'impact des ségrégations territoriales et universitaires sur les parcours étudiants : la filière AES », Formation Emploi, n 120(4), pp. 57-74.

Orange S. (2013), "Interroger le choix des études supérieures ", Genèses, n 89(4), pp. 112-127.

Robette N. (2011), Explorer et décrire les parcours de vie : les typologies de trajectoires, Paris, CEPED, «Les Clefs pour... », 85 p.

Robette N., Thibault N. (2008), "L'analyse exploratoire de trajectoires professionnelles : analyse harmonique qualitative ou appariement optimal ? ", Population, n 64(3), pp. 621-646.

Rohwer G., Pötter U. (2005), TDA user's manual, T Software, Ruhr-Universität Bochum.

Sarfati F. (2015), «Faut-il être compétent pour pouvoir développer ses compétences ? L'exemple de la sélection à l'entrée en Master $2 »$, Formation Emploi, n 130, pp. 31-48.

Studer M., Ritschard G., Gabadinho A., Müller N. (2011), "Discrepancy Analysis of State Sequences”, Sociological Methods \& Research, n 40(3), pp. 471-510.

Truong F. (2015), « De la Zep aux ECTS. Les carrières étudiantes des lycéens de SeineSaint-Denis ", in Courty C. (dir.), La mobilité dans le système scolaire, une solution pour la réussite et la démocratisation, Lille, Presses Universitaires du Septentrion, pp. 73-92. 